\title{
The Effect of Infra-Slow Fluctuation Neurofeedback Training on a Cohort of Insomnia Participants
}

\author{
Melissa Bekker ${ }^{1}$, Karlien Balt ${ }^{3}$, Priyesh Bipath', Joyce Jordaan², and Peet du Toit ${ }^{1}$ \\ ${ }^{1}$ Department of Physiology, Faculty of Health Sciences, University of Pretoria, Prinshof, South Africa \\ ${ }^{2}$ Department of Statistics, Faculty of Natural and Agricultural Sciences, University of Pretoria, Hatfield, South Africa \\ ${ }^{3}$ Karlien Balt Neurofeedback, Lynnwood Manor, Pretoria, South Africa
}

\begin{abstract}
Neurofeedback has gained great interest as a noninvasive treatment for various disorders. However, there is still a lack in literature regarding the effects of infra-slow fluctuation (ISF) neurofeedback training. ISF neurofeedback training is aimed at the lowest brainwave oscillations and attempts to balance dysregulated brainwave activity by inducing shifts in the parasympathetic and sympathetic response. The aim of this study was to determine whether ISF neurofeedback training has a significant effect in participants with insomnia by using quantitative electroencephalography (qEEG), Central Nervous System Vital Signs (CNS VS), and by measuring the changes of physiological vitals. The intervention consisted of 10 sessions for 40 participants separated equally into two groups. Significant results were achieved with improved core temperature $(p=.002)$, finger temperature $(p$ $<.001)$, lower heart rate $(p=.002)$, systolic $(p=.003)$ and diastolic blood pressure $(p=.001)$. The qEEG components significantly improved within standard ranges. An improved neurocognitive state was achieved in terms of CNS VS, with a decrease in depression $(p=.003)$, anxiety $(p<.001)$, and stress $(p<.001)$. This study demonstrated that ISF neurofeedback training should be considered as a viable alternative that can be used concurrently with other insomnia treatment methods.
\end{abstract}

Keywords: insomnia, ISF, neurofeedback, qEEG, CNS Vital Signs, ANS vitals

Citation: Bekker, M., Balt, K., Bipath, P., Jordaan, J., \& du Toit, P. (2021). The effect of infra-slow fluctuation neurofeedback training on a cohort of insomnia participants. NeuroRegulation, 8(3), 137-148. https://doi.org/10.15540/nr.8.3.137

*Address correspondence to: Melissa Bekker, MSc Physiology, Department of Human Physiology, Faculty of Health Sciences, University of Pretoria, Pretoria, South Africa, 0181. Email: bekker.melissa@gmail.com

Copyright: (c) 2021. Bekker et al. This is an Open Access article distributed under the terms of the Creative Commons Attribution License (CC-BY).

\section{Edited by:}

Rex L. Cannon, PhD, SPESA Research Institute, Knoxville, Tennessee, USA

\section{Reviewed by:}

Rex L. Cannon, PhD, SPESA Research Institute, Knoxville, Tennessee, USA

Randall Lyle, PhD, Mount Mercy University, Cedar Rapids, lowa, USA

\section{Introduction}

Sleep is associated with distinct and important physiological changes; however, it is often thought of as a nonactive phase during which individuals become rested. On the contrary, sleep is essential for general health and well-being and is a highly regulated, active process which involves various metabolic pathways (Carley \& Farabi, 2016).

Insomnia typically occurs when a metabolic pathway becomes dysregulated. Insomnia is categorized as a sleep disorder by the International Classification of Sleep Disorders (ICSD-3; Judd \& Sateia, 2019), as well as the fifth edition of the Diagnostic and
Statistical Manual of Mental Disorders (DSM-5; American Psychiatric Association [APA], 2013). Insomnia is characterized by the inability to fall asleep or to stay asleep despite having the opportunity for sleep, which results in daytime dysfunction. Due to inadequate quantity and quality of sleep, individuals present with fatigue, varying levels of irritability, poor work performance and daytime functional impairments (Devi et al., 2018). There are numerous models that describe the pathophysiology of insomnia, and include physiological hyperarousal; genetic, molecular, and cellular mechanisms; dysregulated electrophysiology; and behavioral and cognitive factors (Ban et al., 2011). 
The management and treatment of insomnia is divided into nonpharmacological treatments and pharmacological therapies. A nonpharmacological treatment that is often utilized is cognitive behavioral therapy (CBT-I; Krystal et al., 2019). Pharmacological therapies include the use of medications from different classes such as benzodiazepines (Sieghart \& Sperk, 2002), z-drugs (Krystal et al., 2019), melatonin receptor agonists (Mayer et al., 2009; $\mathrm{Ng}$ et al., 2017; Wing et al., 2012), selective H1 antagonists (Krystal et al., 2013), orexin receptor antagonists (Michelson et al., 2014), antidepressants (Krystal, 2009), antipsychotics (Krystal, 2009), nonselective antihistamines (Morin et al., 2005), and anticonvulsants (Gajraj, 2007; Krystal et al., 2019; Rose \& Kam, 2002). Unfortunately, pharmacological therapies have associated risks and side effects which may result in drug dependency.

In an attempt to reduce pharmacological dependence, researchers have started to investigate alternative treatment methods such as neurofeedback. Neurofeedback is a modality of biofeedback that is focused on the brain and is based on scientific concepts developed in the 1950s to elicit conscious control over the autonomic nervous system (Demos, 2005). It is a noninvasive, nonpharmacological method that is used to alter underlying cognitions and behaviors based on brainwave abnormalities by using self-regulation (Enriquez-Geppert et al., 2017). Self-regulation of the brain's wave patterns is learned through operant conditioning (Asher \& Wierbowski, 2017).

Neurofeedback encourages operant conditioning by pairing desirable and undesirable brainwave activity with a feedback signal such as visual and auditory feedback (Marzbani et al., 2016). Feedback is based on electrophysiological components, and rewards or punishments are awarded accordingly. Rewards are provided when the desired brain activity is achieved and is in the form of an auditory tone and continuation of visual video content. Punishments are provided when undesired brain activity occurs and is in the form of high or low auditory tones and dimming of visual video content (Marzbani et al., 2016).

The type of neurofeedback that was used in this research study is infra-slow fluctuation (ISF) neurofeedback training. ISF neurofeedback training is directed at the lowest frequencies that the brain produces, known as $<0.1 \mathrm{~Hz}$ (Smith, 2018b). The purpose of ISF training is to find the optimal frequency at which the subjects' brainwaves are most balanced. This state of homeostasis refers to a state in which dysregulated brainwave characteristics such as amplitude, coherence, and deviancy of brainwave activity are improved within standard ranges and represent a balanced model of the brain as seen in a quantitative electroencephalography (qEEG) report.

ISF training relies on cortical activation and induces shifts between the sympathetic and parasympathetic response (Peché, 2015). Previous research has demonstrated that ISFs originate from mechanisms at a local cellular level, mainly from neurons, glia, and blood components (Palva \& Palva, 2012). Together, these ISF potentials reflect the working of underlying physiological networks by forming a superstructure of ISFs that regulate, integrate, and initiate decoupling between neuronal networks that are active. Therefore, during an ISF neurofeedback session this superstructure of neuronal networks is addressed and targeted by modification of the shared information associated between cortical areas (Smith, 2013).

Each ISF neurofeedback session results in slow signal reinforcement within the brain which further produces behavioral changes and physiological improvements (Smith, 2013). By training the lowest energy levels of the brain, ISF neurofeedback can potentially coordinate the processes of the autonomic nervous system such as heart rate, blood pressure, blood flow, and digestion, and achieve a mental balance between calmness and being alert (Smith, 2018a). The reinforcement of regulated brain states promotes the readjustment of brainwave activity in various regions of the brain, and more towards the standardized baseline of activity levels (Smith, 2018a). After readjustment, the brain becomes more flexible to change to ensure appropriate responses to situations and the environment that can induce a fight-or-flight or restand-digest activation (Smith, 2018a).

However, ISF neurofeedback has only recently gained significant research interest; therefore, scientific publications and evidence of the mechanisms thereof are still scarce. Published ISF neurofeedback research areas include the effects thereof in ADHD, obesity, food addictions, anxiety, autism, and chronic pain (Balt et al., 2020; Collura \& Frederick, 2016; Leong et al., 2018; Pigott \& Cannon, 2014). Therefore, ISF neurofeedback was identified as the intervention method during this study, to demonstrate whether its effects can be applied to sleep disorders due to growing demands for alternative treatments. 


\section{Methods}

This research study was approved by the Faculty of Health Sciences, Masters and Ethics Committee (63/2020) of the University of Pretoria. Informed consent was obtained from all of the participants that were recruited. Participants were provided with a review session during which their results were discussed.

\section{Aim}

The aim of this study was to determine whether ISF neurofeedback training had a significant effect on qualitative electroencephalogram activity, CNS Vital Signs, and physiological vitals in participants with insomnia.

\section{Participant Criteria and Selection}

The research study was designed as a quasiexperimental study with a control group. A total sample size of 40 participants was recruited. Participants were assigned to an experimental group $(n=20)$ or a control group $(n=20)$. Participants recruited for the experimental group were based on reports and diagnosis of insomnia by a clinical neurophysiologist or medical professional; whereas, the control group had to meet the criteria of being between the ages of 18 and 75, as well as agree out of their own volition to do neurofeedback training as required for the study. Participants were excluded if they had epilepsy, changed medication whilst doing ISF training, reported use of recreational drugs, or if participants were pregnant at the time of recruitment.

\section{Pre and Post Measurements}

Electrophysiological Properties. Participants of both the experimental and control group underwent a comprehensive 21-channel qEEG, pre and post of the 10 ISF neurofeedback sessions. The qEEG was completed with the BrainMaster Discovery 24 Series (BrainMaster Technologies, Inc., Bedford, $\mathrm{OH}$ ), using sintered $\mathrm{AgCl} / \mathrm{Cl}$ electrodes together with Ten20 conductive paste and Nuprep scrub to ensure the adhesiveness of the electrodes to the scalp. The international 10-20 system was followed for accurate electrode placement. The software that was utilized included Brain Avatar version 4.6.4. and qEEG-Pro, which provided an in-depth analysis of EEG components of interest such as: $z$-scores, deviant voxels, amplitude, and coherence (qEE-Pro, Netherlands).

Neurocognitive Function. A Central Nervous System Vital Signs (CNS VS) assessment was completed online pre and post of the 10 ISF neurofeedback sessions to determine and evaluate the neurocognitive states of all participants (CNS Vital Signs, 2019). The assessment consisted of 12 standardized tests assigned to the sleep protocol. The tests and questionnaires included the verbal memory test, visual memory test, finger tapping test, symbol digit coding, Stroop test, shifting attention test, continuous performance test, pain scale test (PST), Epworth Sleepiness Scale (ESS), Pittsburgh Sleep Quality Index (PSQI), Medical Outcomes Survey (MOS) and the Depression, Anxiety and Stress scale (DASS; CNS Vital Signs, 2019).

Physiological Parameters. To determine the effect of ISF neurofeedback training within a single session, physiological vitals such as blood pressure, heart rate, core and finger temperature measurements were obtained pre and post each ISF neurofeedback session, for all participants.

To improve the accuracy of the reading, blood pressure and heart rate measurements were obtained from the left arm twice, pre and post each ISF neurofeedback session. Measurements were obtained using a Clicks Extra-large Blood Pressure Monitor (Clicks, Cape Town, South Africa).

Pre and post of each ISF neurofeedback session, participants were instructed to hold the sensor of the Stress Thermometer SC911 between their thumb and index finger for $30 \mathrm{~s}$ to obtain a finger temperature measurement (Bio-Medical Instruments, Inc., Clinton Township, MI). Core temperature measurements were made by the placement of the sensor underneath the arm in the armpit for 30 seconds.

\section{ISF Neurofeedback}

For the purposes of this research study, ISF neurofeedback training required the use of 5 sintered $\mathrm{Ag} / \mathrm{AgCl}$ electrodes (Peché, 2018). The electrodes were connected to the BrainMaster Atlantis I (4 x 4) amplifier via a 2-channel input cable (BrainMaster Technologies, Inc., Bedford OH). The software that was used to set the training frequency was Brain Avatar version 4.6.4. A screen/monitor and a sound system were used to display video and audio, respectively. The audio of the movie together with the different reward and punishment tones were used for the ISF training. The active electrodes were placed at the T3/T4 sites on the scalp and are commonly used as the starting point for ISF neurofeedback training, which allows for training across the hemispheres. Furthermore, there were two reference electrodes placed behind each ear on 
the mastoid bone and one ground electrode placed in the centre of the scalp, known as $\mathrm{Cz}$.

The training was initiated at a frequency of 0.0030 $\mathrm{Hz}$ and if participants demonstrated or reported any physiological changes the frequency was changed to either a higher or lower frequency depending on whether the symptom reflected activation of the sympathetic nervous system or the parasympathetic nervous system, in increments of $0.0005 \mathrm{~Hz}$.

The auditory feedback was specific to each participant as it guided their brain toward the set frequency to achieve an overall balance of the brainwaves. The visual feedback was provided with a "Dimmer Window." This dimmer screen dimmed the screen during neurofeedback training to provide a secondary feedback. The screen dimmed if the brainwaves fluctuated away from the desired baseline and encouraged the waves towards a balanced and regulated state.

The ISF training session duration was $30 \mathrm{~min}$ in total, thereafter the participant had to report whether they experienced any symptoms after the training, within the first 24 hours. During every subsequent training session, shifts in frequency were made until a constant state of positive symptoms had been achieved at their determined optimum frequency and if the brainwaves were demonstrating improvement and stabilization in terms of EEG components.

Figure 1. Research and experimental design.

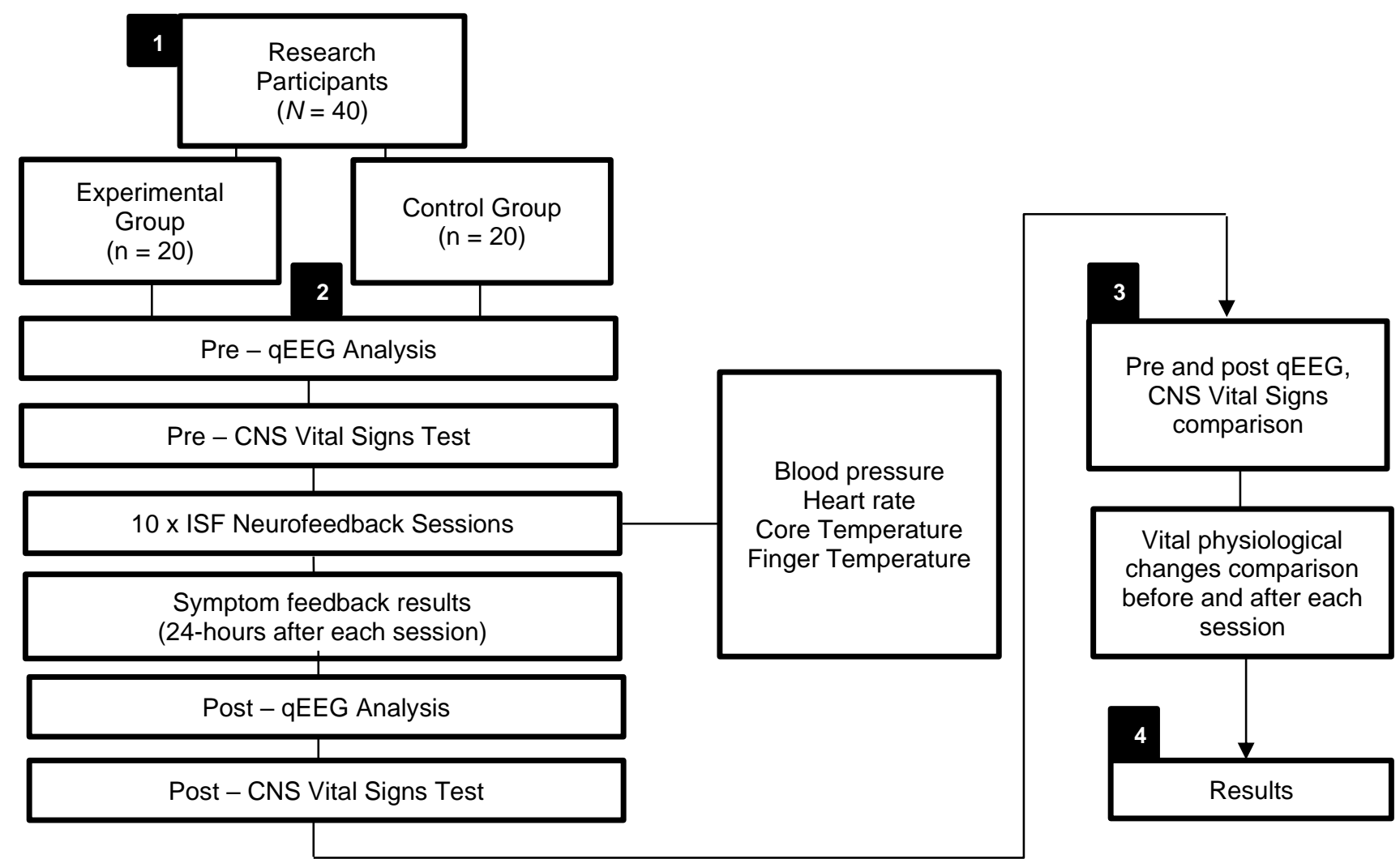

\section{Statistical Analysis}

IBM SPSS Statistics version 26 was utilized to conduct the statistical analysis. The data obtained were analyzed by conducting descriptive statistical analysis together with nonparametric tests such as the Related-Samples Wilcoxon Signed Rank test. A comparison was completed between the groups for each measured component by an IndependentSamples Mann-Whitney $U$ test to determine if the groups responded in the same or different manner in response to the ISF neurofeedback training and to eliminate potential placebo effects.

\section{Results}

The experimental group $(n=20)$ consisted of 11 females and 9 males, with a mean age of 34 years. 
The control group $(n=20)$ consisted of 12 females and 8 males, with a mean age of 27 years.

\section{Physiological Vitals}

Table 1 represents the Related-Samples Wilcoxon Signed Rank test summaries and the significance level for the control and experimental groups by calculating the median difference of the measured vitals. Figure 2 (Graphs A-E) illustrates the mean differences achieved in both groups that are measured pre and post from the ISF neurofeedback training sessions.

\begin{tabular}{|c|c|c|c|c|}
\hline \multicolumn{5}{|c|}{$\begin{array}{l}\text { Table } 1 \\
\text { Vital Test Summaries }\end{array}$} \\
\hline \multicolumn{5}{|c|}{ Related-Samples Wilcoxon Signed Rank Test Summary } \\
\hline \multirow{6}{*}{ 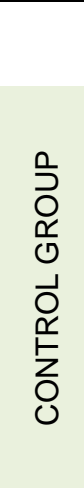 } & Vitals & $\begin{array}{c}\text { Test } \\
\text { Statistic }\end{array}$ & $\begin{array}{l}\text { Standard } \\
\text { Error }\end{array}$ & $\begin{array}{c}\text { Significance } \\
(p \text {-value })\end{array}$ \\
\hline & $\begin{array}{l}\text { Core } \\
\text { Temperature }\end{array}$ & 4.000 & 26.786 & $<0.001$ \\
\hline & $\begin{array}{c}\text { Finger } \\
\text { Temperature }\end{array}$ & 0.000 & 26.786 & $<0.001$ \\
\hline & Heart rate & 180.500 & 26.782 & 0.005 \\
\hline & $\begin{array}{l}\text { Systolic blood } \\
\text { pressure }\end{array}$ & 170.000 & 26.782 & 0.015 \\
\hline & $\begin{array}{l}\text { Diastolic blood } \\
\text { pressure }\end{array}$ & 145.500 & 28.847 & 0.042 \\
\hline \multirow{5}{*}{ 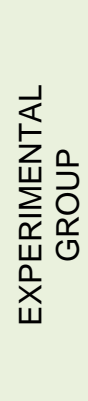 } & $\begin{array}{c}\text { Core } \\
\text { Temperature }\end{array}$ & 20.000 & 26.786 & 0.002 \\
\hline & $\begin{array}{c}\text { Finger } \\
\text { Temperature }\end{array}$ & 1.000 & 26.786 & $<0.001$ \\
\hline & Heart rate & 186.000 & 26.786 & 0.002 \\
\hline & $\begin{array}{l}\text { Systolic blood } \\
\text { pressure }\end{array}$ & 185.000 & 26.786 & 0.003 \\
\hline & $\begin{array}{l}\text { Diastolic blood } \\
\text { pressure }\end{array}$ & 198.000 & 26.784 & 0.001 \\
\hline
\end{tabular}

Both groups achieved significant $p$-values for all measured vitals that represent the change that occurred in the autonomic physiological vital functioning. The change in core and finger temperature demonstrate an increase in temperature after ISF neurofeedback training. This result illustrates a change in blood circulation based on stress levels. When a participant is stressed or tensed, blood circulation is directed to major organs and muscles due to vasoconstriction of blood vessels beneath the skin. Therefore, hands and feet are often cold when a participant is in a stressed state and a drop in temperature can be noted when measured at the extremities. When a participant is more relaxed, peripheral blood circulation is increased due to vasodilation of blood vessels and hands and feet become warmer. Therefore, an increase in the measured temperatures from the extremities can be expected (Bio-Medical Instruments, Inc., Clinton Township, MI).

A significant change was achieved for heart rate in both groups. This difference represents an overall decline in heart rate from pre-post measurements. Therefore, participants demonstrated a lower heart rate after ISF neurofeedback training.

Figure 2. Mean pre and post values obtained for core temperature, finger temperature, heart rate, systolic blood pressure and diastolic blood pressure from the control and experimental group during the course of 10 ISF neurofeedback sessions.
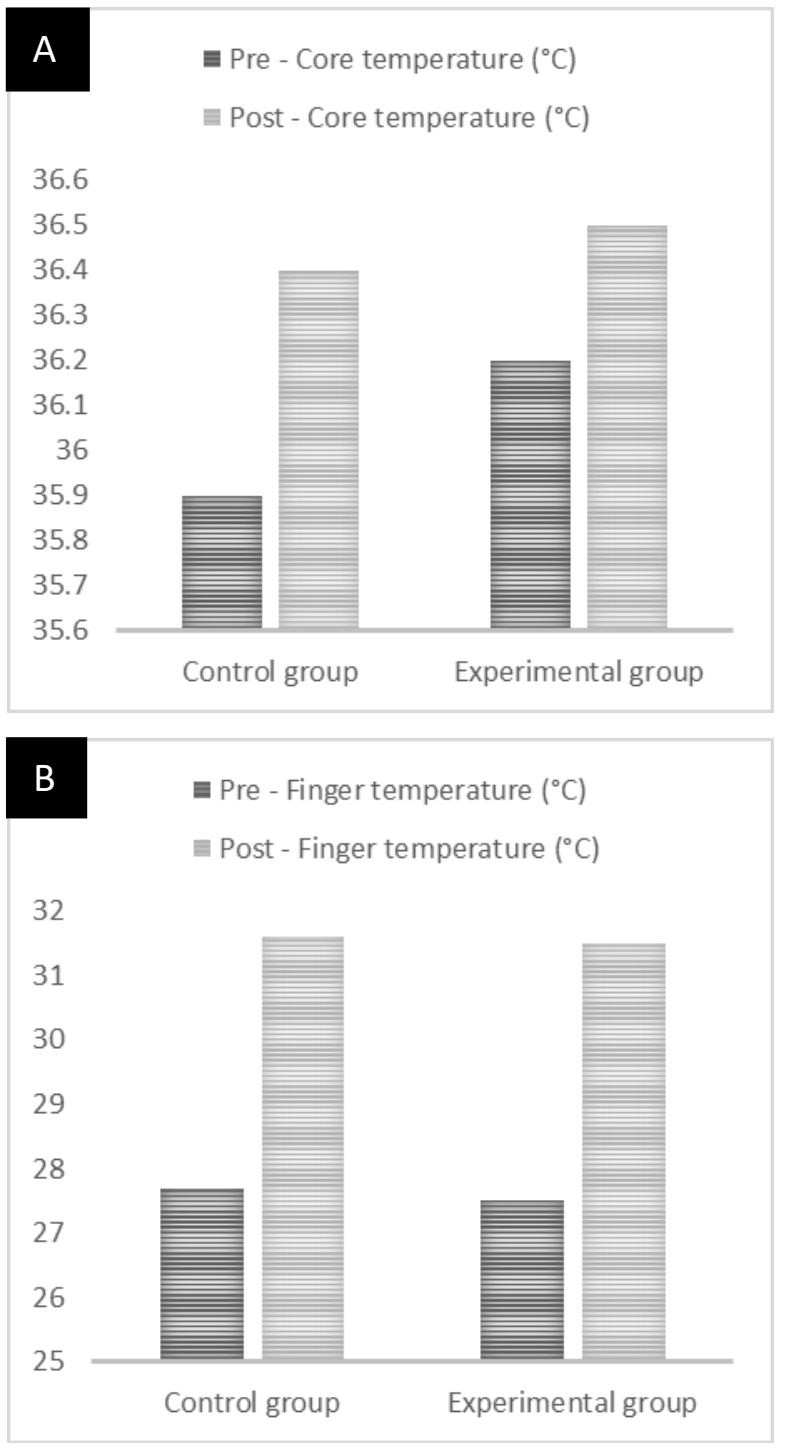


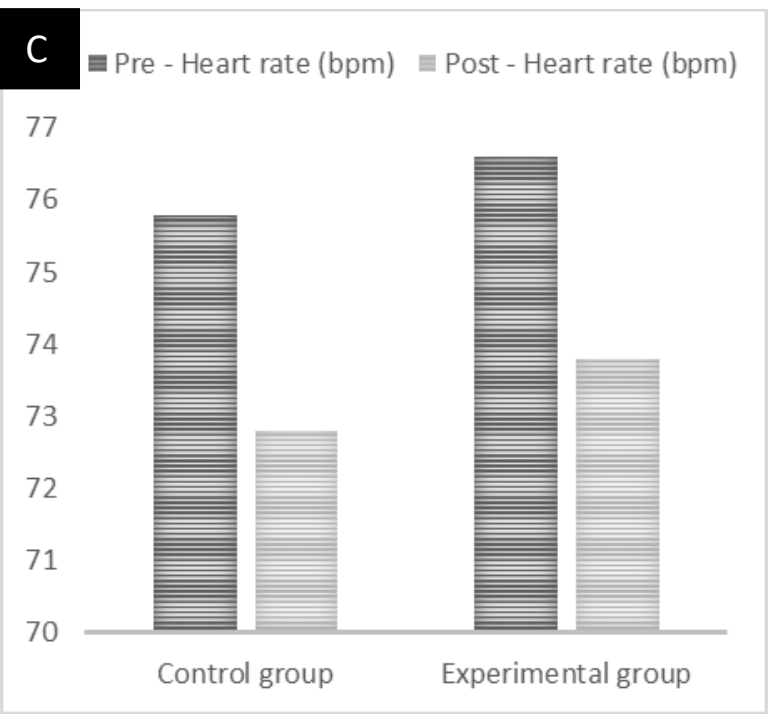

\section{D $\quad$ E Pre - Systolic blood pressure $(\mathrm{mmHg})$ \\ E Post - Systolic blood pressure $(\mathrm{mmHg})$}

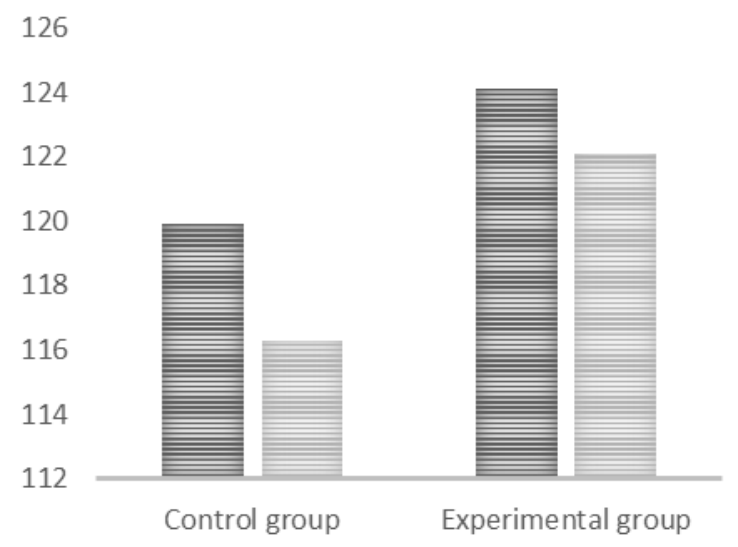

$\mathbf{E} \quad$ = Pre - Diastolic blood pressure $(\mathrm{mmHg})$

E Post - Diastolic blood pressure $(\mathrm{mmHg})$

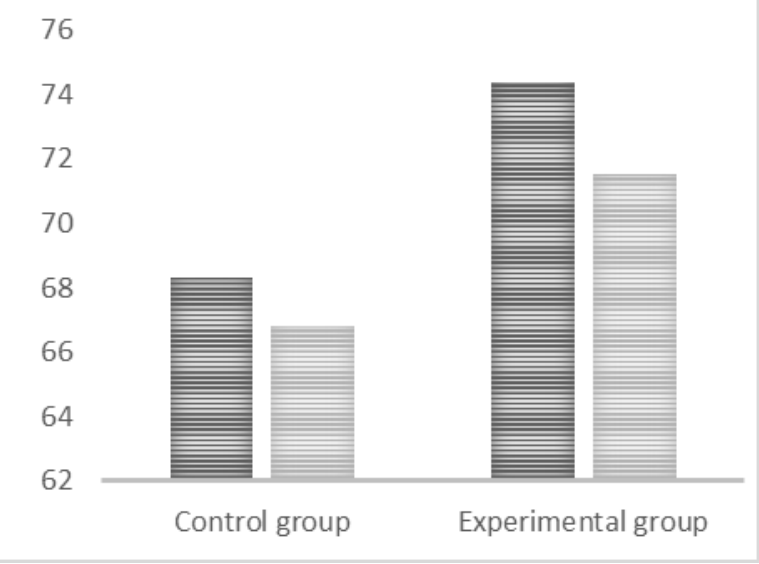

An overall decrease in systolic blood pressure was achieved from pre-post for both groups. A significant decrease in the diastolic blood pressure was also achieved. However, participants from the experimental group demonstrated higher prediastolic blood pressure in comparison to the control group. This difference in prediastolic blood pressure supports the insomnia pathophysiology model regarding altered blood pressure measurements which can result in an increased arousal state and ultimately insomnia symptoms (Zhang et al., 2014).

\section{The qEEG Components}

The qEEG components were analyzed pre-post in both eyes-open and eyes-closed states for each component separately, namely z-scores, deviant voxels, amplitude, and coherence. Previous EEG research studies concluded that eyes-open and eyes-closed conditions should be used as separate baseline resting states due to fluctuating dominancy of brainwaves in each state. During the eyes-open state, alpha brainwave activity is suppressed due to visual stimulation within the posterior cortical regions, whereas suppression in the delta and theta brainwave activity has been noted during eyes-open states in frontal and lateral regions of the brain (Kan et al., 2017).

Z-Scores. A z-score represents a standardized value that corresponds with normal or abnormal brainwave activity and is categorized within standard deviations. For this $z$-score analysis, the aim was to determine whether $z$-scores for each brainwave significantly increased or decreased. The Independent-Samples Wilcoxon Signed Rank Test was utilized to determine the significance of the change measured pre-post from the ISF neurofeedback intervention.

\section{Table 2}

Z-Score Eyes-Open (EO) and Eyes-Closed (EC) p-values

\begin{tabular}{lrrrr}
\hline & \multicolumn{2}{c}{ Control Group } & \multicolumn{2}{c}{ Experimental Group } \\
Brainwave & $p(\mathrm{EO})$ & $p(\mathrm{EC})$ & $p(\mathrm{EO})$ & $p(\mathrm{EC})$ \\
Delta & 0.001 & 0.881 & $<0.001$ & 0.331 \\
Theta & $<0.001$ & 0.232 & $<0.001$ & 0.219 \\
Alpha & 0.011 & 0.499 & 0.005 & 0.103 \\
Lo-Beta & 0.001 & 0.191 & 0.003 & 0.600 \\
Beta & $<0.001$ & 0.017 & 0.005 & 0.121 \\
Hi-Beta & 0.004 & 0.040 & 0.012 & 0.251 \\
Gamma & 0.012 & 0.029 & 0.011 & 0.067 \\
Alpha1 & 0.012 & 0.469 & 0.011 & 0.192 \\
Alpha2 & 0.011 & 0.385 & 0.027 & 0.571 \\
\hline
\end{tabular}


Significant results were achieved for all brainwave $z$-scores in both groups in the eyes-open state. This finding is indicative of $z$-scores decreasing within normal standard deviations. Significant findings in the eyes-closed state were only achieved for the control group's z-scores of beta $(p=0.017)$, hi-beta $(p=0.040)$ and gamma $(p=0.029)$. Overall, the $z$ scores in the eyes-closed state demonstrated little change, and more investigation is required to determine the full extent of ISF neurofeedback effects in both eye states.

\section{Deviant Voxels}

A deviant voxel represents the deviant percentage of a specific voxel within an array. In this study the brain was modelled as a three-dimensional array by qEEG-Pro and corresponded with 10-20 electrode placements. These placements further provided reference points from which deviant voxels can be measured. The deviant voxels for each brainwave were calculated cumulatively across the brain and the percentage measurement was utilized for analysis. The Independent-Samples Wilcoxon Signed Rank Test was utilized in both eye states to determine the significance of the measured changes in deviant voxels.

As seen in Table 3, significantly improved results were achieved overall and for each brainwave in an eyes-open state in the control group.

\begin{tabular}{|c|c|c|c|c|}
\hline \multicolumn{5}{|l|}{$\begin{array}{l}\text { Table } 3 \\
\text { Deviant }\end{array}$} \\
\hline \multicolumn{5}{|c|}{ Related-Samples Wilcoxon Signed Rank Test Summary } \\
\hline \multirow[b]{2}{*}{ Brainwave } & \multicolumn{2}{|c|}{ Control Group } & \multicolumn{2}{|c|}{ Experimental Group } \\
\hline & $p(\mathrm{EO})$ & $p(E C)$ & $p(\mathrm{EO})$ & $p(\mathrm{EC})$ \\
\hline Overall & $<0.001$ & 0.140 & $<0.001$ & 0.023 \\
\hline Delta & 0.001 & 0.161 & $<0.001$ & 0.100 \\
\hline Theta & 0.001 & 1.000 & $<0.001$ & 0.011 \\
\hline Alpha & 0.003 & 0.546 & 0.005 & 0.779 \\
\hline Lo-Beta & $<0.001$ & 0.390 & 0.001 & 0.019 \\
\hline Beta & 0.002 & 0.104 & 0.001 & 0.007 \\
\hline Hi-Beta & 0.001 & 0.062 & $<0.001$ & 0.007 \\
\hline Gamma & 0.012 & 0.070 & 0.104 & 0.023 \\
\hline Alpha1 & 0.003 & 0.456 & 0.015 & 0.793 \\
\hline Alpha2 & 0.005 & 0.926 & 0.042 & 0.985 \\
\hline
\end{tabular}

These significant findings are indicative of a decrease in deviant voxel percentages. The experimental group displayed a similar trend with significant findings measured overall and in each brainwave except for the deviant voxel measurements of the gamma brainwave.

There were no significant changes in the deviant voxel measurements obtained in an eyes-closed state from the control group. Significant eyes-closed results were achieved in the experimental group for the global overall $(p=0.023)$, theta $(p=0.011)$, lo-beta $(p=0.019)$, beta $(p=0.007)$, hi-beta $(p=$ $0.007)$ and gamma $(p=0.023)$ deviant voxel measurements.

\section{Amplitude and Coherence}

Amplitude refers to the size of the peak of the signal from each brainwave, measured in microvolts. Coherence refers to the similarity of the frequency of two signals and reflects how interconnected two sites in the brain are in terms of the information being shared between the sites (Warner, 2013).

Amplitude and coherence measurements can be standardized into two categories of standard deviations namely, positive $(+1,+2)$ and negative $(-1,-2)$ deviations. The aim of the analysis was to determine if amplitude and coherence measurements shifted in standard deviations and moved towards the norm ( 0 ) by either demonstrating an increase in the first (+/-) standard deviation or a decrease in the second (+/-) standard deviation.

The Related-Samples Wilcoxon Signed Rank Test was used in the analysis to determine the median differences for both $(+/-)$ standard deviations of the amplitude and coherence measurements. As seen in Table 4, significant findings were achieved in the eyes-open state for the control and experimental group in all amplitude and coherence groups except for the category of: Amplitude $\% Z<-1$ and Amplitude $\% Z<-2$. Similar results were achieved in the eyes-closed state with the control and experimental group reporting significant findings for all amplitude and coherence groups except for the category of: Amplitude $\% Z<-1$ and Amplitude $\% Z<$ -2 . This result is due to an overall pre- and postdistribution within the positive $(+1,+2)$ standard deviation categories, demonstrating that participants generally have high amplitude peaks instead of low amplitude peaks that are distributed across the brain.

Coherence was measured to determine if ISF neurofeedback training is effective in improving coherence across all measured 10-20 sites and if there is any correlation with an improvement in insomnia symptoms. As stipulated, significant $p$ - 
values were achieved for both groups in positive and negative standard deviation categories. This result indicates that coherence measurements became more distributed within the standard deviation categories.

\begin{tabular}{|c|c|c|c|c|}
\hline \multicolumn{5}{|c|}{$\begin{array}{l}\text { Table } 4 \\
\text { Amplitude and Coherence } p \text {-values }\end{array}$} \\
\hline \multicolumn{5}{|c|}{ Related-Samples Wilcoxon Signed Rank Test Summary } \\
\hline \multirow[b]{2}{*}{$\begin{array}{l}\text { Amplitude (A) } \\
\text { \& Coherence } \\
\text { (C) \%Z }\end{array}$} & \multicolumn{2}{|c|}{ Control Group } & \multicolumn{2}{|c|}{ Experimental Group } \\
\hline & $p(\mathrm{EO})$ & $p(E C)$ & $p(\mathrm{EO})$ & $p(\mathrm{EC})$ \\
\hline$A \% Z>1$ & $<0.001$ & $<0.001$ & 0.002 & $<0.001$ \\
\hline$A \% Z>2$ & $<0.001$ & $<0.001$ & 0.001 & $<0.001$ \\
\hline$A \% Z<-1$ & 0.465 & 1.000 & 1.000 & 0.258 \\
\hline$A \% Z<-2$ & 1.000 & 1.000 & 0.180 & 1.000 \\
\hline$C \% Z>1$ & $<0.001$ & $<0.001$ & 0.019 & 0.010 \\
\hline$C \% Z>2$ & 0.000 & $<0.001$ & 0.012 & 0.009 \\
\hline$C \% Z<-1$ & $<0.001$ & $<0.001$ & 0.019 & 0.002 \\
\hline$C \% Z<-2$ & $<0.001$ & $<0.001$ & 0.013 & 0.001 \\
\hline
\end{tabular}

CNS Vital Signs

CNS Vital Signs were utilised to determine neurocognitive function pre and post of the ISF neurofeedback intervention. As seen demonstrated in Table 5, CNS VS component results were reported as standardized values and represent a level of neurocognitive function. The significance of the change measured from the standardized score was correlated with a $p$-value.

Neurofeedback Symptoms

The statistical analysis of the neurofeedback symptoms involved the analysis of the symptoms reported by the participants on the neurofeedback symptom report. Symptoms measured included emotional reactivity, emotional sensitivity, difficulty falling asleep, lack of deep sleep, agitation, sedation, physical tension, dizziness, nightmares, sugar cravings, appetite, headaches, nausea, diarrhea, and constipation.

This analysis consisted of a cross-tabulation of the total count of reported cases for each symptom, the percentage thereof, together with the standardized residual value. The degree of the symptom was ranked in a range of $1-3$, with $1=$ no symptom, $2=$ mild symptom, and $3=$ severe symptom.

\begin{tabular}{|c|c|c|}
\hline \multicolumn{3}{|l|}{$\begin{array}{l}\text { Table } 5 \\
\text { CNS Vital Signs } p \text {-values }\end{array}$} \\
\hline \multicolumn{3}{|c|}{ Related-Samples Wilcoxon Signed Rank Test Summary } \\
\hline CNS Vital Signs Component & $\begin{array}{l}\text { Control } \\
\text { Group } p\end{array}$ & $\begin{array}{l}\text { Experimental } \\
\text { Group } p\end{array}$ \\
\hline Neurocognition Index (NI) & 0.002 & 0.005 \\
\hline Composite Memory & 0.197 & 0.670 \\
\hline Verbal Memory & 0.289 & 0.687 \\
\hline Visual Memory & 0.214 & 0.777 \\
\hline Psychomotor speed & 0.016 & 0.409 \\
\hline Reaction Time & 0.141 & 0.030 \\
\hline Complex Attention & 0.115 & 0.028 \\
\hline Cognitive Flexibility & 0.031 & 0.002 \\
\hline Processing Speed & 0.026 & 0.153 \\
\hline Executive Function & 0.021 & 0.002 \\
\hline Simple Attention & 0.054 & 0.598 \\
\hline Motor Speed & 0.765 & 0.708 \\
\hline Pain Scale & 0.014 & 0.010 \\
\hline $\begin{array}{l}\text { Epworth Sleepiness Scale } \\
\text { (ESS) }\end{array}$ & 0.182 & 0.214 \\
\hline Duration of Sleep & 0.102 & 0.011 \\
\hline Sleep Latency & 0.007 & 0.005 \\
\hline Sleep Efficiency & 0.131 & 0.022 \\
\hline Need Meds to Sleep & 0.317 & 0.039 \\
\hline Sleep Disturbance & 1.000 & 0.008 \\
\hline $\begin{array}{l}\text { Day Disfunction Due to } \\
\text { Sleepiness }\end{array}$ & 0.194 & 0.004 \\
\hline Overall Sleep Quality & 0.166 & $<0.001$ \\
\hline PSQI Total Score & 0.032 & $<0.001$ \\
\hline Physical Functioning & 0.453 & 0.440 \\
\hline Role Functioning - Physical & 0.167 & 0.836 \\
\hline Role Functioning - Emotional & 0.088 & 0.016 \\
\hline Energy/Fatigue & 0.073 & 0.017 \\
\hline Emotional Well-being & 0.070 & 0.014 \\
\hline Social Functioning & 0.005 & 0.107 \\
\hline Pain & 0.289 & 0.716 \\
\hline General Health & 0.137 & 0.265 \\
\hline Health Change & 0.928 & 0.175 \\
\hline Depression & 0.007 & 0.003 \\
\hline Anxiety & 0.005 & $<0.001$ \\
\hline Stress & 0.027 & $<0.001$ \\
\hline
\end{tabular}


Most of the symptoms that were reported were within the first degree of the scale. Therefore, the severity of the symptoms experienced after ISF neurofeedback training were low with only certain symptoms being reported as severe, namely emotional reactivity $(10 \%)$, difficulty falling asleep $(30 \%)$, lack of deep sleep $(30 \%)$, sugar cravings $(10 \%)$, and diarrhea (10\%) across both groups.

\section{Discussion}

Participants achieved improved regulation of ANS vitals as seen with the measurement results. Core temperature post measurements became distributed between $36.5^{\circ} \mathrm{C}$ and $37^{\circ} \mathrm{C}$ for both groups and demonstrated improved regulation in comparison to pre-measurements which were scattered between $34^{\circ} \mathrm{C}$ and $37.5^{\circ} \mathrm{C}$. Finger temperature measurements demonstrated both groups becoming more relaxed and improved regulation with shifts towards the parasympathetic response with post measurements ranging between $30^{\circ} \mathrm{C}$ and $34^{\circ} \mathrm{C}$. Pre measurements achieved lower peaks in temperature between $25^{\circ} \mathrm{C}$ and $30^{\circ} \mathrm{C}$ and demonstrated that participants were likely to be more tense before the ISF neurofeedback training.

Heart rate post measurements decreased within ranges of $60-80$ beats per minute for both groups, whereas pre measurements ranged between 70-90 beats per minute. Therefore, heart rate became more regulated after ISF neurofeedback training with healthier ranges which further demonstrated improved relaxation of participants. Post measurements of systolic blood pressure demonstrated ranges between $115-130 \mathrm{mmHg}$ for both groups, in comparison to higher pre measurement ranges of $120-140 \mathrm{mmHg}$. Furthermore, diastolic blood pressure post measurements reached a peak at $70 \mathrm{mmHg}$ for both groups, whereas pre measurements were scattered between $60-80 \mathrm{mmHg}$. Therefore, improved cardiovascular activity was achieved in terms of heart rate and blood pressure measurements for both groups. This outcome further demonstrated that ISF neurofeedback training can elicit a calming effect and improve vital function regulation in participants.

The significant results achieved for core and finger temperature, heart rate, systolic and diastolic blood pressure are substantial as this confirms that ISF neurofeedback training impacts the regulation of autonomic functions of the ANS. Furthermore, these significant results support the findings of previous neurofeedback research in which researchers demonstrated that ISF neurofeedback training can regulate ANS functions by using peripheral biofeedback indicators (Balt et al., 2020).

These ANS vital findings are of great significance as they provide a pathway for ISF neurofeedback training as a potential cotreatment for insomnia, as some models of pathophysiology suggest that individuals can develop insomnia due to a comorbidity such as elevated blood pressure or abnormal temperature regulation.

Within the qEEG analysis, $z$-scores measured after the ISF neurofeedback intervention were significantly lower in both groups. This decrease in $z$-score values demonstrate that ISF neurofeedback training has the ability to train $z$-scores within standardized brainwave activity ranges and establishes a balanced overall state across all brainwaves.

Significant findings were achieved for the deviant voxels for all brainwaves in the eyes-open state in the control and experimental group. No significant findings were achieved in the eyes-closed state for the control group. Significant results were achieved for only specific brainwaves in the experimental group, namely global overall, theta, lo-beta, beta, hibeta and gamma. A plausible reason for a significant change in deviant voxel activity in the previously mentioned brainwaves for the experimental group is that these brainwaves are mainly responsible for initiating slow wave activity (theta) that induces sleep and faster brainwave activity (lo-beta, beta, hibeta, gamma) that is required for day-to-day functioning. In the experimental group these brainwaves may be initially dysregulated as described in the pathophysiology model of dysregulated electrophysiology and creates symptoms of insomnia (Ban et al., 2011; Perlis et al, 2001).

Other qEEG components that play an important role with regards to the balance achieved between the various brainwaves is amplitude and coherence. Amplitude can be trained across brainwave bandwidths to improve asymmetries during ISF neurofeedback training. The measured amplitude results support the theory of hyperarousal and dysregulated electrophysiological properties associated with insomnia (Ban et al., 2011; Riemann et al., 2010). The analysis further demonstrated that amplitudes decreased from pre to post measurements. Amplitudes decreased within standard deviations and moved towards standardized norms for each participant's age. 
Therefore, amplitude measurements were distributed across the brain within standardized ranges after the ISF neurofeedback training intervention.

Coherence refers to the interconnectivity and synchronization of the frequency of two signals between two sites in the brain (Warner, 2013). This measurement can be used to determine if regions in the brain are in a state of hypo- or hypercoherence. Hypocoherence is a state of poor intersite interaction and can result in poor efficiency of cognitive processes due to cortical regions being unable to connect and share information between sites; whereas hypercoherence refers to a rigid state where patterns of intersite connections become locked together. This results in the brain becoming dependant on the intersite patterns and ultimately only activating certain cortical structures and centers. The brain then becomes unable to process information efficiently and obstructs the formation of new neural connections (Warner, 2013).

As stipulated, significant $p$-values were achieved for both groups in positive and negative standard deviation categories. This result indicates that coherence measurements became more distributed within the standard deviation categories. Thus, coherence measurements improved after the ISF neurofeedback intervention in both eye states with the control group reporting more instances of hypercoherence and the experimental group both hyper- and hypocoherence.

CNS Vital Signs provided in-depth neurocognitive function reports of all participants pre and post of the ISF neurofeedback intervention. Significant $p$-values were achieved for various neurocognitive components as listed, but most importantly significant results were achieved for both groups in the Pittsburgh Sleep Quality Index (PSQI). Thus, both groups reported improved quality of sleep after 10 sessions of ISF neurofeedback training. Furthermore, significant results were achieved for both groups in the Depression, Anxiety and Stress Score (DASS) test. Demonstrating improvements in depression, anxiety, and stress. The DASS results support previous research findings suggesting that ISF neurofeedback has the potential to be utilized as long-term treatment plans for depression, anxiety, and stress (Smith et al., 2014).

To further improve the quality of scientific reporting, placebo effects were determined between the experimental and control group. All measured components were compared between the groups to determine if participants displayed similar trends in the effects of the ISF neurofeedback training. The Independent-Samples Mann-Whitney $U$ test was used to statistically analyze the trends between both groups. No significant differences were achieved between the groups after the ISF neurofeedback training intervention took place. Therefore, no placebo effects were reported in this study, as both groups statistically displayed the same responses in effects after ISF neurofeedback training.

In summary, the aim together with all objectives set out in the research protocol were achieved. Importantly, participants demonstrated improved qEEG and CNS Vital Signs outcomes with an overall improvement in the quality of sleep, ANS vital regulation such as heart rate, body temperature and blood pressure, as well as improved neurocognitive functions and a decrease in depression, anxiety, and stress. Therefore, ISF neurofeedback training has demonstrated its efficacy in improving qEEG activity together with neurocognitive factors which can aid in the improvement of insomnia symptoms in an insomnia cohort.

\section{Conclusion}

This study demonstrated that by using ISF neurofeedback training to improve vitals, neurocognitive functions and dysregulated electrophysiological brain activity, insomnia symptoms can be improved. Therefore, ISF neurofeedback training should be considered as a viable supplementary insomnia treatment that can be used concurrently with other treatment methods, which can reduce the dependence of pharmacological treatments. ISF neurofeedback training has demonstrated remarkable results in various areas of research and future research studies should continue to aim to include a variety of scientific principles to ensure that the validity of ISF neurofeedback training is upheld.

\section{Acknowledgements}

Karlien Balt, for providing access to the KB Neurofeedback cc practice, facilities, and apparatus. CNS Vital Signs, for providing discounted research rates for the online assessments.

\section{Author Declarations}

A bursary was provided by the postgraduate funding department of the University of Pretoria, for the tuition fees associated with the research study. The authors declare that there is no conflict of interests involved in this research study. 


\section{References}

American Psychiatric Association. (2013). Diagnostic and statistical manual of mental disorders (5th ed.). https://doi.org/10.1176/appi.books.9780890425596

Asher, T., \& Wierbowski, B. (2017). Brain training: The future of psychiatric treatment? Harvard University. https://sitn.hms.harvard.edu/flash/2017/brain-training-futurepsychiatric-treatment/

Atlantis I 4x4 series system [Apparatus]. (2020). Bedford, OH: BrainMaster Technologies, Inc. https://brainmaster.com /product/atlantis/

Balt, K., Du Toit, P., Smith, M. L., \& van Rensburg, C. J. (2020). The effect of infraslow frequency neurofeedback on autonomic nervous system function in adults with anxiety and related diseases. NeuroRegulation, 7(2), 64-74. https://doi.org/10.15540/nr.7.2.64

Ban, H-J., Kim, S. C., Seo, J., Kang, H-B., \& Choi, J. K. (2011). Genetic and metabolic characterization of insomnia. PLoS ONE, 6(4), e18455. https://doi.org/10.1371 /journal.pone.0018455

BrainAvatar 4.0 Discovery Acquisition (Version 4.6.4) [Computer software]. (2019). Bedford, OH: BrainMaster Technologies Inc. https://www.brainmaster.com/product/brainavatar-4-0discovery-acquisition/

BrainMaster Discovery 24 Series [Computer software and hardware]. (2019). Bedford, OH: BrainMaster Technologies, Inc.

Carley, D. W., \& Farabi, S. S. (2016). Physiology of sleep. Diabetes Spectrum, 29(1), 5-9. https://doi.org/10.2337 /diaspect.29.1.5

CNS Vital Signs. (2019). CNS Vital Signs Interpretation Guide. Morrisville, NC: CNS Vital Signs, LLC. https://www.cnsvs.com WhitePapers/CNS VS BriefInterpretation Guide.pdf

Collura, T. F., \& Frederick, J. A. (2016). Handbook of clinical QEEG and neurotherapy. New York, NY: Routledge/Taylor \& Francis Group.

Demos, J. N. (2005). Getting started with neurofeedback. W. W. Norton \& Company.

Devi, C. B. P., Samreen, S., Kumari, N. K., \& Sharma, J. (2018). A review on insomnia: The sleep disorder. The Pharma Innovation Journal, 7(12), 227-230. https://www.thepharmajournal.com/archives/2018/vol7issue1 2/PartE/7-11-125-807.pdf

Enriquez-Geppert, S., Huster, R. J., \& Hermann, C. S. (2017). EEG-neurofeedback as a tool to modulate cognition and behavior: A review tutorial. Frontiers in Human Neuroscience, 11, 51. https://doi.org/10.3389/fnhum.2017.00051

Gajraj, N. M. (2007). Pregabalin: Its pharmacology and use in pain management. Anesthesia \& Analgesia, 105(6), 18051815. https://doi.org/10.1213/01.ane.0000287643.13410.5e

Judd, B. G., \& Sateia, M. J. (2019). Classification of sleep disorders. https://www.uptodate.com/contents/classificationof-sleep-disorders\#H4

Kan, D. P. X., Croarkin, P. E., Phang, C. K., \& Lee, P. F. (2017). EEG differences between eyes-closed and eyes-open conditions at the resting stage for euthymic participants. Neurophysiology, 49(6), 432-440. https://doi.org/10.1007 /s11062-018-9706-6

Krystal, A. D. (2009). A compendium of placebo-controlled trials of the risks/benefits of pharmacological treatments for insomnia: The empirical basis for U.S. clinical practice. Sleep Medicine Reviews, 13(4), 265-274. https://doi.org/10.1016 /j.smrv.2008.08.001

Krystal, A. D., Prather, A. A., \& Ashbrook, L. H. (2019). The assessment and management of insomnia: An update. World Psychiatry, 18(3), 337-352. https://doi.org/10.1002 wwps.20674

Krystal, A. D., Richelson, E., \& Roth, T. (2013). Review of the histamine system and the clinical effects of $\mathrm{H}_{1}$ antagonists:
Basis for a new model for understanding the effects of insomnia medications. Sleep Medicine Reviews, 17(4), 263272. https://doi.org/10.1016/j.smrv.2012.08.001

Large screen arm blood pressure monitor [Apparatus]. (2020). Capetown, South Africa: Clicks. https://clicks.co.za /clicks large-screen-arm-bloodpressuremonitor/p/300191? gclid=CjwKCAiA2O39BRBjEiwApB2lkq98ieHRx5YYmuehv4U IvaaD3GA9J-ZZ35VbjHMPxDeXg5CWSPI8NBoCgc8QAvD_ BwE\&gclsrc $=a w . d s$

Leong, S. L., Vanneste, S., Lim, J., Smith, M., Manning, P., \& De Ridder, D. (2018). A randomised, double-blind, placebocontrolled parallel trial of closed-loop infraslow brain training in food addiction. Scientific Reports, 8, 11659. https://doi.org /10.1038/s41598-018-30181-7

Marzbani, H., Marateb, H. R., \& Mansourian, M. (2016). Neurofeedback: A comprehensive review on system design, methodology and clinical applications. Basic and Clinical Neuroscience, 7(2), 143-158. https://doi.org/10.15412 IJ.BCN.03070208

Mayer, G., Wang-Weigand, S., Roth-Schechter, B., Lehmann, R., Staner, C., \& Partinen, M. (2009). Efficacy and safety of 6month nightly ramelteon administration in adults with chronic primary insomnia. Sleep, 32(3), 351-360. https://doi.org /10.1093/sleep/32.3.351

Michelson, D., Snyder, E., Paradis, E., Chengan-Liu, M., Snavely, D. B., Hutzelmann, J., Walsh, J. K., Krystal, A. D., Benca, R. M., Cohn, M., Lines, C., Roth, T., \& Herring, W. J. (2014). Safety and efficacy of suvorexant during 1-year treatment of insomnia with subsequent abrupt treatment discontinuation: $A$ phase 3 randomised, double-blind, placebo-controlled trial. The Lancet Neurology, 13(5), 461-471. https://doi.org /10.1016/S1474-4422(14)70053-5

Morin, C. M., Koetter, U., Bastein, C., Ware, J. C., \& Wooten, V. (2005). Valerian-hops combination and diphenhydramine for treating insomnia: A randomized placebo-controlled clinical trial. Sleep, 28(11), 1465-1471. https://doi.org/10.1093/sleep 28.11.1465

Ng, K. Y., Leong, M. K, Liang, H., \& Paxinos, G. (2017). Melatonin receptors: Distribution in mammalian brain and their respective putative functions. Brain Structure and Function, 222(7), 2921-2939. https://doi.org/10.1007/s00429-017-1439-

Palva, J. M., \& Palva, S. (2012). Infra-slow fluctuations in electrophysiological recordings, blood-oxygenation-leveldependent signals, and psychophysical time series. Neurolmage, 62(4), 2201-2211. https://doi.org/10.1016 /j.neuroimage.2012.02.060

Peché, D. (2015). ISF or Infra Slow Fluctuation Training. Neurofeedback Therapy. http://www.neurofeedbacktherapy.co.za/Wordpress/2015/02/26/isf-or-infra-slowfrequency-training/

Perlis, M. L., Smith, M. T., Andrews, P. J., Orff, H., \& Giles, D. E. (2001). Beta/gamma EEG activity in patients with primary and secondary insomnia and good sleeper controls. Sleep, 24(1), 110-117. https://doi.org/10.1093/sleep/24.1.110

Pigott, H. E., \& Cannon, R. (2014). Neurofeedback is the best available first-line treatment for ADHD: What is the evidence for this claim? NeuroRegulation, 1(1), 4-23. https://doi.org 110.15540/nr.1.1.4

qEEG-Pro [Database]. (2019). Netherlands: qEEG-Pro B. V. https://qeegpro.eegprofessionals.nl/database/

Riemann, D., Spiegelhalder, K., Feige, B., Voderholzer, U., Berger, M., Perlis, M., \& Nissen, C. (2010). The hyperarousal model of insomnia: A review of the concept and its evidence. Sleep Medicine Reviews, 14(1), 19-31. https://doi.org /10.1016/j.smrv.2009.04.002

Rose, M. A., \& Kam, P. C. A. (2002). Gabapentin: Pharmacology and its use in pain management. Anaesthesia, 57(5), 451462. https://doi.org/10.1046/j.0003-2409.2001.02399.x 
Sieghart, W., \& Sperk, G. (2002). Subunit composition, distribution and function of GABA(a) receptor subtypes. Current Topics in Medicinal Chemistry, 2(8), 795-816. https://doi.org/10.2174/1568026023393507

Sintered Silver/Silver Chloride Electrodes. (2019). https://www.eeginfoeurope.com/fileadmin/images/produkte/el ektroden/sintered-electrodes.pdf

Smith, M. L. (2013). Infra-slow fluctuation training; On the downlow in neuromodulation. NeuroConnections, Fall 2013, 38-46.

Smith, M. (2018a). The benefits of infraslow neurofeedback training. Neurofeedback Services of New York. https://neurofeedbackservicesny.com/the-benefits-ofinfraslow-neurofeedback-training/

Smith, M. (2018b). Types of neurofeedback. Neurofeedback Services of New York. https://neurofeedbackservicesny.com /types-of-neurofeedback/

Smith, M. L., Collura, T. F., Ferrera, J., de Vries, J. (2014). Infraslow fluctuation training in clinical practice: A technical history. NeuroRegulation, 1(2), 187-207. https://doi.org /10.15540.nr.1.2.187

Stress Thermometer SC911 [Apparatus]. (2020). Clinton Township, MI: Bio-Medical Instruments, Inc. https://biomedical.com/stress-thermometer-sc911.htm
Warner, S. (2013). Cheat Sheet for Neurofeedback. Stress Therapy Solutions. http://www.stresstherapysolutions.com /uploads//STSCheatSheetoftheBrain.pdf

Wing, Y. K., Zhang, J., Lam, S. P., Li, S. X., Tang, N. L., Lai, K. Y., \& Li, A. M. (2012). Familial aggregation and heritability of insomnia in a community-based study. Sleep Medicine, 13(8), 985-990. https://doi.org/10.1016/j.sleep.2012.04.013

Zhang, J., Lam, S.-P., Li, S. X., Ma, R. C. W., Kong, A. P. S., Chan, M. H. M., Ho, C-S., Li, A. M., \& Wing, Y.-K. (2014). A community-based study on the association between insomnia and hypothalamic-pituitary-adrenal axis: Sex and pubertal influences. The Journal of Clinical Endocrinology \& Metabolism, 99(6), 2277-2287. https://doi.org/10.1210 /jc.2013-3728

Received: August 30, 2021

Accepted: September 2, 2021

Published: September 30, 2021 